\title{
Comprehensive Analysis and Validation Reveal Potential MYCN Regulatory Biomarkers Associated with Prognosis of Neuroblastoma
}

Bo Chen ( $\nabla$ bchen@cmu.edu.cn )

Shengjing Hospital of China Medical University https://orcid.org/0000-0001-8118-2405

\section{Research Article}

Keywords: Neuroblastoma, MYCN, Biomarkers, Prognosis, Targeting Molecules

Posted Date: January 7th, 2022

DOI: https://doi.org/10.21203/rs.3.rs-1202163/v1

License: (c) (i) This work is licensed under a Creative Commons Attribution 4.0 International License.

Read Full License 


\section{Abstract \\ Background}

Neuroblastoma (NB) is an embryonic malignant tumor that occurs in the sympathetic nervous system.

The treatment effect of patients in the high-risk group is poor, and relapse and treatment failure can occur even with multiple combination treatments. MYCN is an independent prognostic factor for $\mathrm{NB}$, and the proportion of MYCN amplification in tumor tissues of high-risk patients reaches $40-50 \%$. Hence, exploring new MYCN targeting molecules is a meaningful way to treat high-risk NB patients.

\section{Methods}

The microarray datasets were obtained from Gene Expression Omnibus, and differentially expressed genes were identified. Gene Ontology, Kyoto Encyclopedia of Genes and Genomes, and miRPathDB were used for function and pathway analysis. The STRING and Cytoscape were used to construct a proteinprotein interaction network and modular analysis. The miRNet and NetworkAnalyst databases were used to predict and construct target gene-miRNA and target gene-TF networks. The R2 database was used for expression, correlation, and prognosis analysis. The diagnostic effect of the biomarkers was predicted by ROC analysis, and rt-PCR was used to verify the hub genes. Finally, used specific siRNA and plasmid to knockdown and overexpressed MYCN, the mRNA levels were verified.

\section{Results}

We identified 431 DEGs and FBX09, HECW2, MIB2, RNF19B, RNF213, TRIM36, and ZBTB16 as novel biomarkers that affect the prognosis of the NB patients. In addition, FBX09, RNF19B, and TRIM36 were preliminarily confirmed to be potential targeting molecules of MYCN.

\section{Conclusions}

Our results are expected to become novel biomarkers for the treatment of high-risk NB patients.

\section{Introduction}

Neuroblastoma (NB) is the most common extracranial solid malignant tumor in children[1]. It predisposes to the adrenal medulla but can also occur in the sympathetic nervous system of the neck, chest, and abdomen[2, 3]. NB usually occurs in younger children, with a median age of 17 months at diagnosis[4-6]. Its heterogeneity characterizes NB, and tumors can metastasize without treatment and develop resistance to therapy[7]. Its clinical manifestations range from asymptomatic masses to primary tumors caused by local invasion or widespread diseases[8]. According to reports, the incidence of NB in childhood cancer is 
second only to leukemia and brain tumors, accounting for about $8 \%$ of childhood malignancies and $15 \%$ of all childhood cancer deaths[4].

Studies have shown that the prognosis of NB patients is related to factors such as age at diagnosis, tumor stage, and oncogene MYCN amplification $[9,10]$. According to the clinical manifestations and the biological characteristics of the tumor, NB patients can be divided into a low-risk group, a medium-risk group, and a high-risk group[6]. Among them, the age of onset of patients in the low and moderate risk groups is generally within 1.5 years of age, and the lesions are mostly localized. Generally, there is less amplification of MYCN, so the response to surgery and chemotherapy is better[11]. In some patients with low risk and good biological characteristics, self-healing can often be achieved, and the long-term survival rate can reach more than $90 \%[12]$. The age of onset of patients in the high-risk group is mostly over 1.5 years old, and they generally have manifestations of MYCN expansion[6]. Extensive metastatic lesions often appear at the time of diagnosis, even in the case of intensive chemotherapy combined with surgery, radiotherapy, and autologous bone marrow stem cell transplantation. The long-term survival rate is still less than $40 \%[10]$.

MYCN is a member of the MYC proto-oncogene family, and the prognosis of tumor patients is poor when overexpressed[9]. MYCN can promote tumor progression by inducing cell proliferation, dedifferentiation, and mitochondrial metabolism disorders[13]. According to reports, about $20 \%$ of the tumor tissues of NB patients have MYCN amplification, and the proportion of MYCN amplification in tumor tissues of highrisk patients is as high as $40-50 \%$. MYCN is an independent prognostic factor for NB patients[14, 15]. Studies have shown that molecular markers were related to the occurrence and development of tumors and can be used for early tumor screening. However, the problem is that the expression of molecular markers in tumors does not have reasonable specificity[16]. Hence, it is necessary to explore new MYCN targeting molecules further to provide new strategies for diagnosing and treating high-risk NB patients.

This study downloaded the GEO database's microarray datasets GSE12460, GSE13136, GSE14880, GSE16237, and GSE73517. The differentially expressed genes (DEGs) were screened by comparing the gene expression between MYCN amplified and MYCN non-amplified NB cells. STRING and Cytoscape were used to construct protein-protein interaction (PPI) networks and perform modular analysis of DEGs. Then, Gene Ontology (GO), Kyoto Encyclopedia of Genes and Genomes (KEGG), and miRPathDB were used to perform function and pathway analysis, miRNet and NetworkAnalyst were used to construct a target gene-miRNA regulatory and target gene-TF regulatory network. The R2 database was used to confirm the relationship between the genes and MYCN Correlation, and the importance of prognosis has determined FBX09, HECW2, MIB2, RNF19B, RNF213, TRIM36, and ZBTB16 as hub genes. From the microarray dataset GSE73517 to verify the results were reliable, the diagnostic effect of the hub genes was predicted by Receiver Operator Characteristic Curve (ROC) analysis. Finally, the use of cell transfection technology to knockdown or overexpression MYCN through PCR experiments preliminarily confirmed that MYCN regulates the expression of hub genes FBX09, RNF19B, and TRIM36. In conclusion, this study attempted to explore promising new targeting molecules for MYCN that predicts the prognosis 
of NB. Targeting FBX09, RNF19B, and TRIM36 in NB cells may represent a novel strategy for NB therapeutic intervention.

\section{Materials And Methods}

\section{Acquisition of Microarray Data}

Five microarray datasets (GSE12460, GSE13136, GSE14880, GSE16237, and GSE73517) were obtained from the GEO (http://www.ncbi.nlm.nih.gov/geo/) database. The GSE12460 contains 14 MCYN amplification and $32 \mathrm{MYCN}$ non-amplification NB cells[17], GSE13136 contains 10 MCYN amplification and 20 MYCN non-amplification NB cells[18], GSE14880 contains 12 MCYN amplification and 18 MYCN non-amplification NB cells[19], GSE16237 contains 7 MCYN amplification and 44 MYCN nonamplification NB cells[20], GSE73517 contains 33 MCYN amplification and 72 MYCN non-amplification NB cells[21]. Data from GSE73517 were used for validation and based on the GPL16876 platform (Agilent-020382 Human Custom Microarray 44k, Feature Number version). The other microarray datasets were based on the GPL570 platform (Affymetrix Human Genome U133 Plus 2.0 Array).

\section{Identification of differentially expressed genes}

Based on the Affy package in the R language (http://cran.r-project.org/), the robust multi-array average (RMA) algorithm converts the original microarray dataset into expression values and then performs background correction and quintiles Normalization and probe summary. The paired t-test of the limma package of the R language was used to analyze the DEGs between the MYCN-amplification and the MYCN non-amplification NB cells. The volcanos were generated using the ggplot2 package of the $\mathrm{R}$ language, and Benjamini-Hochberg's method was used to control the False Discovery Rate (FDR). P-value $<0.05$ and $|\log 2 \mathrm{FC}|>1$ were the critical values for screening DEGs.

\section{GO and KEGG enrichment analysis of DEGs}

Use the online tool Venny ( $v$ 2.1, https://bioinfogp.cnb.csic.es/tools/venny/) to construct a Venn diagram to identify overlapping modules and genes in the DEGs of the four microarray datasets. DAVID (http://david.abcc.ncifcrf.gov/) is an annotation, visualization, and comprehensive discovery database. It is an online tool for gene function classification used to analyze and evaluate biological gene functions[22]. miRPathDB ( $v$ 2.0, https://mpd.bioinf.uni-sb.de) is a new dictionary on miRNAs and target pathways, and the database contains many miRNAs, different miRNA target sets, and a wide range of functional biochemical categories[23]. Here, we used the DAVID database to perform GO and KEGG enrichment analysis to study the function of DEGs, and miRPathDB was used for pathway verification of miRNAs. $P<0.05$ was regarded as the critical point with statistical significance.

\section{PPI network construction and module analysis}

STRING ( 11.5 , https://string-db.org) is an online tool to search for interacting genes and proteins. DEGs were entered into the database to construct a PPI network that shows physical and functional 
interactions. In this study, proteins with a score (minimum required interaction score) $>0.9$ were selected for PPI network construction. In addition, Cytoscape (v 3.8.2, https://cytoscape.org) was used to visualize the network, calculate the node degree through Network Analyzer, and draw the PPI network with different colors and sizes to show the adjustment and node degree. The MCODE plug-in (degree cutoff $=2$, node score cutoff $=0: 2$, and $\mathrm{K}$ - core $=2$ ) was used to identify the hub modules in the network, and the BiNGO plug-in and DAVID were used to analyze the function and pathway enrichment of the genes in the hub modules, which can better explain the reliability of the results. $P<0.05$ was used as the critical value for screening.

\section{miRNA prediction of target genes}

miRNet (v 2.0, https://www.mirnet.ca/) database was used to predict the miRNA associated with the target genes, construct a target gene-miRNA interaction network, and Cytoscape was used to visualize it to characterize further the relationship between the target genes and miRNAs Correlation.

\section{Transcription factor prediction of target genes}

NetworkAnalyst (v 3.0, https://www.networkanalyst.ca/) is a series of network-based tools for statistical meta-analysis, visual data mining, and integration through the rapid generation of biological networks. It supports meta-analysis of gene lists, and data integration is achieved through robust statistical procedures, followed by visual inspection in the PPI network[24]. Used NetworkAnalyst to predict the Transcription Factor (TF) of the target genes to characterize the correlation between the potential target genes and TFs.

\section{Analysis of Hub Genes}

The R2 database (http://r2platform.com) is a genomics analysis and visualization platform that provides a biologist-friendly interface for high-throughput data. It was developed in the Cancer Genomics Department of $A M C$ in the Netherlands, where it remains the main entry point for all types of highthroughput data. Here, we screened the DEGs, through the survival analysis of gene expression in related tumors through the R2 database, detected the relationship between their expression in NB and the prognosis of the patient and its correlation with $\mathrm{MYCN}$, and generated related survival curves and charts.

\section{Cell culture and transfection}

NB cell lines: SK-N-AS (AS) and SK-N-BE2 (BE2) used in the present study were kindly provided by Dr. Carol J Thiele from Pediatric Oncology Branch, National Cancer Institute, National Institutes of Health, USA. AS cells is a MYCN non-amplified cell line, while BE2 cells is a MYCN amplified cell line. Used RPMI 1640 culture medium containing $10 \%$ fetal bovine serum, cell culture Cultivated in an incubator at $37^{\circ} \mathrm{C}$, $5 \% \mathrm{CO} 2$, and saturated humidity. Passaged for 2-3 days. Cells in the logarithmic growth phase were used in the experiment. 
The cells were digested with ordinary trypsin, and the cell suspension containing $1.5 \times 10^{5} \mathrm{cell} / \mathrm{s} / \mathrm{ml}$ was inoculated in a 6 -well plate $(2 \mathrm{ml} /$ well), and the cell density during transfection was $50 \%-60 \%$. Washed the cells twice with PBS, added RPMI 1640 medium without double antibodies (penicillin and streptomycin), small interfering RNAs (MYCN siRNA, siRNA control) (Tongyong, Anhui, China), and MYCN overexpression plasmid (NM_001293228, pDsRed2) -N1 vector, Xhol/Kpnl digestion, negative control plasmid) (Genechem, Shanghai, China) was used to transfect NB cells. The target sequences of siRNAs were as follows: MYCN siRNA CGGAGATGCTGCTTGAGAA, used jetPRIME transfection reagent for transfection, refer to the instructions for operation. After 24 hours of transfection, the cells were collected to extract RNA, and RT-PCR detected the mRNA level.

\section{Real-time quantitative PCR}

The total RNA was extracted with the kit reagent (Invitrogen), the RNA was $5 \mu \mathrm{g}$, and the GoScript ${ }^{\mathrm{TM}}$ reverse transcription system kit (Promega, UK) was used. The SYBR premix Ex Taq ${ }^{\text {TM }}$ II (TaKaRa Clontech) was used following the manufacturer's instructions, and the CDNA after reverse transcription was used for qPCR. The 2(- $\Delta \Delta \mathrm{Ct})$ method was used for gene expression analysis. The primers were used to detect the target genes (Table 1). All primers were purchased from Sangon Biotech (Shanghai, China). The PCR reaction program was as follows: $95^{\circ} \mathrm{C} 30 \mathrm{~s}, 95^{\circ} \mathrm{C} 5 \mathrm{~s}, 60^{\circ} \mathrm{C} 34 \mathrm{~s}$, a total of 40 cycles. Use the $\Delta \Delta \mathrm{Ct}$ method for analysis, the target genes mRNA $=2-\Delta \Delta \mathrm{Ct}$.

\section{Statistical Analysis}

Use GraphPad 9 software for statistical analysis. The data were expressed as mean \pm standard deviation $(\overline{\mathrm{x}} \pm \mathrm{SD})$. The t-test method was used to compare the data between the two groups. $\mathrm{P}<0.05$ was considered statistically significant.

\section{Results}

\section{Microarray datasets identification of DEGs}

To identify DEGs, we performed background correction and normalization processing on the NB microarray datasets GSE12460, GSE13136, GSE14880, and GSE16237. Filtered DEGs through the limma package in the $\mathrm{R}$ language, with $\mathrm{P}$-value $<0.05$ and $|\log 2 \mathrm{FC}|>1$ as the filtering conditions. Among them, the GSE12460 obtained a total of 1479 DEGs, including 402 up-regulated and 1077 down-regulated DEGs, the GSE13136 obtained a total of 2778 DEGs, including 1223 up-regulated and 1555 downregulated DEGs, the GSE14880 obtained a total of 2068 DEGs, including 535 up-regulated and 1533 down-regulated DEGs, GSE16237 obtained a total of 3842 DEGs, including 496 up-regulated and 3346 down-regulated DEGs. The volcanos were used to show the differential expression of genes in the microarray datasets (Fig 1A).

\section{Identification of overlapping DEGs}


To identify the overlapping DEGs, we used the tool Venny to analyze the four microarray datasets and took the overlapping part of the four microarray datasets. In this study, we identified a total of 431 overlapping DEGs, including 90 up-regulated DEGs and 341 down-regulated DEGs (Fig 1B).

\section{GO and KEGG enrichment of overlapping DEGs}

To further understand the functions and pathways involved in overlapping DEGs, we used the DAVID database for enrichment analysis. The results showed that in Biological Processes (BP), DEGs were significantly enriched in cell adhesion, peripheral nervous system development, extracellular matrix organization, muscle organ development, and cell proliferation, in Cellular Components (CC), DEGs Significantly enriched in the neuromuscular junction, neuron projection, cell surface, filopodium, and axon, regarding Molecular Function (MF), DEGs were significant in collagen binding, glycosaminoglycan binding, structural constituent of muscle, receptor binding, actin-binding Enrichment. In addition, KEGG analysis showed that DEGs were significantly enriched in Proteoglycans in cancer, cell adhesion molecules, MicroRNAs in cancer, Glycine, serine, and threonine metabolism, and Axon guidance (Fig 1C).

\section{PPI network construction and module analysis}

To establish the protein-protein interaction, we used the STRING database to construct a PPI network for overlapping DEGs, involving a total of 194 nodes and 401 edges (Fig 2A). The non-interacting proteins were filtered out by Cytoscape, and the algorithm analysis of MCODE was performed, and the module with the highest MCODE score $=9.0$ was selected as the hub module (Fig 2B). Enrichment analysis through BiNGO plug-in showed that DEGs in the module were mainly enriched in ligase activity, zinc ion binding, acid-amino acid ligase activity, ligase activity, forming carbon-nitrogen bonds, transition metalion binding, male germline stem cell division, asymmetric cell division, leg morphogenesis, and ubiquitin ligase complex. The visualization results are shown in Fig 3A. In addition, KEGG enrichment analysis showed that these DEGs were related to Acute myeloid leukemia, Transcriptional misregulation in cancer, MicroRNAs in cancer, and Pathways in cancer. The visualization results are shown in Fig 3B.

\section{miRNA prediction of target genes}

To predict the miRNA related to the target genes, we used the miRNet database to perform predictive analysis, imported the prediction results into Cytoscape for visualization, and constructed a target genemiRNA interaction network (Fig 4). The results showed that FBXO9 interacts with 42 miRNAs (for example, hsa-mir-329-3p), FBX017 interacts with 40 miRNAs (for example, hsa-mir-124-3p), HECW2 interacts with 43 miRNAs (for example, hsa-mir-19a-3p), MIB2 interacts with 17 miRNAs (for example, hsa-mir-335-5p), RNF19B interacts with 117 miRNAs (for example, hsa-mir-17-5p), RNF213 interacts with 140 miRNAs (for example, hsa-mir-101-3p), TRIM36 interacts with 40 miRNAs (for example, hsa-mir-253p), TRIM71 interacts with 163 miRNAs (for example, hsa-let-7a- 5p), ZBTB16 interacts with 53 miRNAs (for example, hsa-mir-15a-5p). The miRNAs in the network were further ranked by degree method, and the top five miRNAs with the highest scores are shown in Table 2. In addition, we further validated the pathways in which these miRNAs were involved through the miRPathDB database, with $P$-value $<0.05$ as 
the filtering conditions. The results showed that these miRNAs were significantly annotated to MicroRNAs in cancer, mTOR signaling, Proteoglycans in cancer, Pathways in cancer, and TGF-beta Signaling Pathway. Most of the pathways, including the five pathways listed earlier, were supported by experimental validation (especially strong experimental validation). The 20 significant pathways are shown in Table 3 .

\section{Transcription factor prediction of target genes}

To predict the TFs related to the target genes, we used the NetworkAnalyst database to conduct predictive analysis and constructed the target gene-TF interaction network (Fig 5A). The results showed that FBX09 interacts with 38 TFs (for example, KLF16), FBX017 interacts with 13 TFs (for example, ZEB1), MIB2 interacts with 35 TFs (for example, SIX5), RNF19B interacts with 17 TFs (for example, EGR2), RNF213 interacts with 51 TFs (for example, IRF1), TRIM36 interacts with 10 TFs (for example, CBX8), TRIM71 interacts with 19 TFs (for example, SOX5), ZBTB16 interacts with 4 TFs (for example, WT1). Further GO enrichment analysis of TFs in the network showed that BP was mainly enriched in peptidyl-lysine modification, covalent chromatin modification, and histone modification, CC was significantly enriched in transcription regulator complex, RNA polymerase II transcription regulator complex, and transcription repressor complex. MF was mainly involved in DNA-binding transcription activator activity, DNA-binding transcription activator activity, RNA polymerase II-specific and DNAbinding transcription repressor activity, RNA polymerase II-specific. The visualization results are shown in Fig 5B. In addition, KEGG enrichment analysis showed that these TFs were related to Transcriptional misregulation in cancers, TGF-beta signaling pathway, Thyroid hormone signaling pathway. The visualization results are shown in Fig 5C.

\section{Survival analysis of hub genes}

To study the correlation between gene expression and the prognosis of NB patients, we further analyzed the data with a sample size of 649 through the R2 database. Finally, we screened out FBXO9, HECW2, MIB2, RNF19B, RNF213, TRIM36, and ZBTB16 among these eight DEGs. The genes closely related to the patient's prognosis were used as hub genes. Compared with NB patients with relatively low hub genes expression in tumor tissues, NB patients with relatively high hub genes expression have a higher overall survival rate $(P<0.001)(F i g 6)$. We further analyzed the correlation between hub genes expression and MYCN expression in NB tissues. The results showed that the expression of hub genes and MYCN in NB tumor tissues were negatively correlated $(P<0.001)$ (Fig 7A). In addition, the expression of hub genes in MYCN non-amplified tumor tissues was higher than that in MYCN-amplified tumor tissues $(P<0.001)$ (Fig 7B).

\section{Diagnostic Effectiveness of Biomarkers for Neuroblastoma}

To make the results more reliable, we used the microarray dataset GSE73517 to verify. Expression levels for MYCN, FBX09, HECW2, MIB2, RNF19B, RNF213, TRIM36, and ZBTB16 are shown in the heatmap (Fig 8A). Consistent with the above results, FBX09, HECW2, MIB2, RNF19B, RNF213, TRIM36, and ZBTB16 have significantly higher expression in MYCN-nonamplified NB cells $(P<0.01)$ (Fig 8B). We analyzed the 
correlation between seven effective biomarkers and MYCN. Correlation results are shown in Fig 8C. In addition, the microarray dataset GSE73517 was used to verify the diagnostic effectiveness of NB biomarkers through ROC analysis. AUC more than 0.800 was considered to have excellent specificity and sensitivity to diagnose NB. As shown in Fig 8D, the AUC values for FBXO9, HECW2, MIB2, RNF19B, RNF213, TRIM36, and ZBTB16 were 0.864 ( $95 \% \mathrm{Cl}, 0.796 \times 0.932), 0.817(95 \% \mathrm{Cl}, 0.739 \times 0.898), 0.902$ $(95 \% \mathrm{Cl}, 0.832 \times 0.973), 0.896(95 \% \mathrm{Cl}, 0.835 \times 0.958), 0.781$ (95\% Cl, $0.677 \times 0.884), 0.918(95 \% \mathrm{Cl}$, $0.866 \times 0.970)$ and $0.881(95 \% \mathrm{Cl}, 0.812 \times 0.950)$.

\section{Experimental verification of hub genes}

RT-PCR technology was used to determine the mRNA levels of seven hub genes (FBXO9, HECW2, MIB2, RNF19B, RNF213, TRIM36, and ZBTB16) in MYCN non-amplified cell line AS and MYCN amplified cell line BE2. As shown in Fig 9, the seven identified hub genes were all highly expressed in MYCN non-amplified NB cell lines $(P<0.05)$, consistent with the bioinformatics analysis results.

\section{MYCN regulates the expression of FBX09, RNF19B, and TRIM36}

To study whether MYCN regulates the hub genes, we designed MYCN siRNA and overexpression plasmids. MYCN siRNA was transfected into MYCN amplified BE2 cells, and MYCN overexpression plasmid was transfected into MYCN non-amplified AS cells. Then detected the changes in the expression of hub genes at the mRNA levels. The results showed that after MYCN siRNA was transfected into BE2 cells, the expression of FBX09, RNF19B, and TRIM36 increased at the mRNA levels, which were consistent with the predicted results (Fig 10A). After the MYCN overexpression plasmid was transfected into AS cells, the expression of FBX09, RNF19B, and TRIM36 decreased at the mRNA levels (Fig 10B). These data indicated that MYCN has a counter-regulatory effect on the expression of FBXO9, RNF19B, and TRIM36 in NB cells.

\section{Discussion}

NB is the most common extracranial tumor in childhood, which has different clinical manifestations and courses according to the biological characteristics of the tumor[6]. Studies have shown that maintaining proliferation is a fundamental feature of cancer cells[25, 26]. In many types of cancer, this ability can be acquired by the MYC family to promote the growth or abnormal activation of tumor transcription factors $[27,28]$. MYC family member MYCN amplification is a cancer-causing event in high-risk NB development, leading to poor prognosis in NB patients[29]. In addition, abnormal MYCN activation is also associated with the pathogenesis of neuroendocrine cancer in adults, including glioblastoma, small cell lung cancer, and pancreatic cancer[30]. Hence, the MYCN status determined in NB diagnosis is a significant adverse prognostic factor. Studying the role and mechanism of new targeting molecules of MYCN is a critical way to explore the treatment of high-risk NB patients.

Bioinformatics analysis technology has been increasingly used to find new therapeutic targets and diagnostic markers for various cancers in recent years[31-33]. Compared with a single cohort study, a 
multi-cohort study has a lower false-positive rate and false-negative rate[34]. In this study, to reduce the impact of batch effects and increase the credibility of DEGs identification, we selected four microarray datasets from the same platform. Here, we have identified a total of 431 overlapping DEGs, including 90 up-regulated DEGs and 341 down-regulated DEGs. The enrichment analysis at the GO level shows that these DEGs were mainly involved in cell adhesion, extracellular matrix organization, cell proliferation, and receptor binding. In addition, KEGG analysis showed that DEGs were significantly enriched in Proteoglycans in cancer, cell adhesion molecules, and MicroRNAs in cancer. These rich functions and pathways provided insights into the molecular mechanism of NB pathogenesis.

To identify the hub genes in DEGs, we constructed a PPI network, analyzed by the MCODE plug-in in Cytoscape, and selected the highest-rated module in the network as the hub module (including FBXO9, FBX017, HECW2, MIB2, RNF19B, RNF213, TRIM36, TRIM71, and ZBTB16). Based on the hub module, we have carried out an enrichment analysis of genes. The results suggested that these DEGs were closely related to many signaling pathways that regulate the development of cancers (For example, Pathways in cancer, mTOR signaling, and PI3K-AKT Signaling in Cancer), which also provided potential value for further research on hub modules.

In the above study, MicroRNAs in cancer and Transcription misregulation in cancer were significant enrichment results for hub modules. Studies have shown that miRNAs and TFs play an essential regulatory role in developing various cancers, including childhood cancer NB[35-37]. In addition, miRNAs and TFs were reported to play an indispensable role in regulating NB proliferation, apoptosis, invasion, and metastasis[38-42]. Here, we further predicted and analyzed the genes in the hub modules of miRNAs and TFs. In the miRNA prediction analysis, we found FBX09, FBX017, HECW2, MIB2, RNF19B, RNF213, TRIM36, TRIM71, and ZBTB16 interacted with a total of 615 miRNAs. In addition, we further verified through the miRPathDB database that these miRNAs were involved in regulating MicroRNAs in cancer, mTOR signaling, Proteoglycans in cancer, Pathways in cancer, and TGF-beta Signaling Pathway. Using the NetworkAnalyst database for TFs predictive analysis, we found FBX09, FBX017, MIB2, RNF19B, RNF213, TRIM36, TRIM71, and ZBTB16 participated with a total of 187 TFs regulation. Enrichment analysis from TFs in the network shows that these TFs were closely related to Transcriptional misregulation in cancers, TGF-beta signaling pathways, and Thyroid hormone signaling pathway. From enrichment analysis of DEGs and hub modules to miRNAs and TFs enrichment verification, we found that these biomarkers were closely related to various cancer signaling pathways.

In combination with the R2 database, we further evaluated the prognosis of genes expression in NB patients. The hub genes of FBX09, HECW2, MIB2, RNF19B, RNF213, TRIM36, and ZBTB16 were identified as closely related to the high prognosis of NB patients. Some related reports have been made in previous studies. For example, Liu JA et al. pointed out that Fbxo9 plays an essential role in mediating Sox10 in destroying Neurog2 protein and directing neural crest progenitor cell lineage to glial cells[43]. HynesSmith RW et al. pointed out that the lack of FBXO9 enhanced proteasome activity and promoted the aggressiveness of acute myeloid leukemia[44]. Yang $L$ et al. pointed out that the participation of circRNA HECW2 in the non-autophagy effect of ATG5 is related to endothelial-mesenchymal transition[45]. Berko 
ER et al. pointed out that de novo missense variants in HECW2 are related to neurodevelopmental delay and hypotonia[46]. Li X et al. pointed out that MIB2 deficiency inhibits microglia activation and reduces brain damage caused by ischemia[47]. Piccolo $\mathrm{P}$ et al. pointed out that the MIB2 variant that alters the NOTCH signal leads to hypertrabeculation/non-compaction of the left ventricle and is associated with Menetrier-like gastropathy[48]. Di Zhang et al. pointed out that RAB5C, SYNJ1, and RNF19B promote male ankylosing spondylitis by regulating the infiltration of immune cells[49]. Banh RS et al. pointed out that PTP1B controls non-mitochondrial oxygen consumption by regulating RNF213 to promote tumor survival during hypoxia[50]. Wang $X$ et al. pointed out that RNF213 inhibits the carcinogenesis of glioblastoma by affecting the MAPK/JNK signaling pathway[51]. Liang $C$ et al. pointed out that as a new androgen-responsive gene, TRIM36 enhances the anti-androgen effect on prostate cancer by inhibiting the MAPK/ERK signaling pathway[52]. He $\mathrm{J}$ et al. pointed out that the BTB/POZ zinc finger protein ZBTB16 inhibits breast cancer proliferation and metastasis by up-regulating ZBTB28 and antagonizing BCL6/ZBTB27[53]. Xiao GQ et al. pointed out that ZBTB16 is a sensitive and specific marker for detecting metastatic and extragonadal yolk sac tumors[54]. Although FBX09, HECW2, MIB2, RNF19B, RNF213, TRIM36, and ZBTB16 have not been reported in NB, based on their role in tumor regulation, this also provides a potential value for their role in NB to a certain extent.

To study the effectiveness of these biomarkers in diagnostic NB, we used the microarray dataset GSE73517 to verify. Consistent with the above analysis results, FBXO9, HECW2 MIB2, RNF19B, RNF213, TRIM36, and ZBTB16 were significantly higher in MYCN nonamplified NB cells. Correlation analysis found that the seven effective biomarkers (FBX09, HECW2, MIB2, RNF19B, RNF213, TRIM36, and ZBTB16) related to MYCN. In addition, the diagnostic effectiveness of the NB biomarkers was verified by ROC analysis. The results also clearly indicated that FBX09, HECW2, MIB2, RNF19B, RNF213, TRIM36, and ZBTB16 were of excellent specificity and sensitivity to diagnose NB.

To verify the results of bioinformatics analysis, we conducted in vitro experiments. First, we detected the mRNA levels of the hub genes in NB cells. The results showed that FBX09, HECW2, MIB2, RNF19B, RNF213, TRIM36, and ZBTB16 have higher expression in MYCN non-amplified NB cells, which was consistent with our analysis. In addition, MYCN siRNA was transfected into BE2 cells, the expression of FBX09, RNF19B, and TRIM36 was increased at the mRNA levels, which was consistent with the analysis results. After the MYCN overexpression plasmid was transfected into AS cells, the expression of FBXO9, RNF19B, and TRIM36 was decreased at the mRNA levels, which further confirmed our results. At present, although there is no report of a regulatory relationship between FBX09, RNF19B, and TRIM36 and MYCN, the prediction based on the databases and the verification of mRNA levels essentially explained the interaction between FBX09, RNF19B, and TRIM36 and MYCN. In addition, FBXO9, RNF19B, and TRIM36 are expected to become the novel target genes of $\mathrm{MYCN}$, but this requires more experimental data to be confirmed.

\section{Abbreviations}


Neuroblastoma

GEO

Gene Expression Omnibus

DEGs

Differentially Expressed Genes

GO

Gene Ontology

KEGG

Kyoto Encyclopedia of Genes and Genomes

PPI

Protein-Protein Interaction

ROC

Receiver Operator Characteristic Curve

RMA

Robust Multi-array Average

FDR

False Discovery Rate

TF

Transcription Factor

BP

Biological Processes

CC

Cellular Components

MF

Molecular Function.

\section{Declarations}

\section{Acknowledgements}

Not applicable.

\section{Author's contributions}

Bo Chen contributed to the study design, interpretation of the data, the writing of the manuscript, and the submission of the manuscript for publication.

\section{Funding}

The author received no specific funding for this work.

\section{Availability of data and materials}


All relevant data generated by authors are within the manuscript.

\section{Ethical Approval and Consent to participate}

Not applicable.

\section{Consent for publication}

Not applicable.

\section{Competing interests}

The authors declared no conflict of interest.

\section{Authors' information}

${ }^{1}$ Department of Pediatrics, Shengjing Hospital of China Medical University, Shenyang, China,

${ }^{2}$ Medical Research Center, Shengjing Hospital of China Medical University, Shenyang, China.

\section{References}

1. Schulpen, M., et al., Significant improvement in survival of advanced stage childhood and young adolescent cancer in the Netherlands since the 1990s. Eur J Cancer, 2021. 157: p. 81-93.

2. Marshall, G.M., et al., The prenatal origins of cancer. Nat Rev Cancer, 2014. 14(4): p. 277-89.

3. Hoehner, J.C., et al., A developmental model of neuroblastoma: differentiating stroma-poor tumors' progress along an extra-adrenal chromaffin lineage. Lab Invest, 1996. 75(5): p. 659-75.

4. Matthay, K.K., et al., Neuroblastoma. Nat Rev Dis Primers, 2016. 2: p. 16078.

5. Brodeur, G.M. and R. Bagatell, Mechanisms of neuroblastoma regression. Nat Rev Clin Oncol, 2014. 11(12): p. 704-13.

6. Pinto, N.R., et al., Advances in Risk Classification and Treatment Strategies for Neuroblastoma. J Clin Oncol, 2015. 33(27): p. 3008-17.

7. Kholodenko, I.V., et al., Neuroblastoma Origin and Therapeutic Targets for Immunotherapy. J Immunol Res, 2018. 2018: p. 7394268.

8. Maris, J.M., Recent advances in neuroblastoma. N Engl J Med, 2010. 362(23): p. 2202-11.

9. Otte, J., et al., MYCN Function in Neuroblastoma Development. Front Oncol, 2020. 10: p. 624079.

10. Fletcher, J.I., et al., Too many targets, not enough patients: rethinking neuroblastoma clinical trials. Nat Rev Cancer, 2018. 18(6): p. 389-400.

11. Shohet, J.M., Redefining functional MYCN gene signatures in neuroblastoma. Proc Natl Acad Sci U S A, 2012. 109(47): p. 19041-2. 
12. Saletta, F., M.S. Seng, and L.M. Lau, Advances in paediatric cancer treatment. Transl Pediatr, 2014. 3(2): p. 156-82.

13. Montemurro, L., et al., A Novel MYCN-Specific Antigene Oligonucleotide Deregulates Mitochondria and Inhibits Tumor Growth in MYCN-Amplified Neuroblastoma. Cancer Res, 2019. 79(24): p. 61666177.

14. Barone, G., et al., New strategies in neuroblastoma: Therapeutic targeting of MYCN and ALK. Clin Cancer Res, 2013. 19(21): p. 5814-21.

15. Westermark, U.K., et al., The MYCN oncogene and differentiation in neuroblastoma. Semin Cancer Biol, 2011. 21(4): p. 256-66.

16. Gao, Y., et al., Multiplex measurement of twelve tumor markers using a GMR multi-biomarker immunoassay biosensor. Biosens Bioelectron, 2019. 123: p. 204-210.

17. Janoueix-Lerosey, I., et al., Somatic and germline activating mutations of the ALK kinase receptor in neuroblastoma. Nature, 2008. 455(7215): p. 967-70.

18. Lastowska, M., et al., Identification of candidate genes involved in neuroblastoma progression by combining genomic and expression microarrays with survival data. Oncogene, 2007. 26(53): p. 743244.

19. Bourdeaut, F., et al., Cholinergic switch associated with morphological differentiation in neuroblastoma. J Pathol, 2009. 219(4): p. 463-72.

20. Ohtaki, M., et al., A robust method for estimating gene expression states using Affymetrix microarray probe level data. BMC Bioinformatics, 2010. 11: p. 183.

21. Henrich, K.O., et al., Integrative Genome-Scale Analysis Identifies Epigenetic Mechanisms of Transcriptional Deregulation in Unfavorable Neuroblastomas. Cancer Res, 2016. 76(18): p. 5523-37.

22. Dennis, G., Jr., et al., DAVID: Database for Annotation, Visualization, and Integrated Discovery. Genome Biol, 2003. 4(5): p. P3.

23. Kehl, T., et al., miRPathDB 2.0: a novel release of the miRNA Pathway Dictionary Database. Nucleic Acids Res, 2020. 48(D1): p. D142-D147.

24. Xia, J., M.J. Benner, and R.E. Hancock, NetworkAnalyst-integrative approaches for protein-protein interaction network analysis and visual exploration. Nucleic Acids Res, 2014. 42(Web Server issue): p. W167-74.

25. Hanahan, D. and R.A. Weinberg, Hallmarks of cancer: the next generation. Cell, 2011. 144(5): p. 64674.

26. Evan, G.I. and K.H. Vousden, Proliferation, cell cycle and apoptosis in cancer. Nature, 2001. 411(6835): p. 342-8.

27. Gordan, J.D., C.B. Thompson, and M.C. Simon, HIF and c-Myc: sibling rivals for control of cancer cell metabolism and proliferation. Cancer Cell, 2007. 12(2): p. 108-13.

28. Masso-Valles, D., M.E. Beaulieu, and L. Soucek, MYC, MYCL, and MYCN as therapeutic targets in lung cancer. Expert Opin Ther Targets, 2020. 24(2): p. 101-114. 
29. Ambros, P.F., et al., International consensus for neuroblastoma molecular diagnostics: report from the International Neuroblastoma Risk Group (INRG) Biology Committee. Br J Cancer, 2009. 100(9): p. 1471-82.

30. Rickman, D.S., J.H. Schulte, and M. Eilers, The Expanding World of N-MYC-Driven Tumors. Cancer Discov, 2018. 8(2): p. 150-163.

31. Riley, R.D., et al., A systematic review of molecular and biological tumor markers in neuroblastoma. Clin Cancer Res, 2004. 10(1 Pt 1): p. 4-12.

32. Brady, S.W., A.M. Gout, and J. Zhang, Therapeutic and prognostic insights from the analysis of cancer mutational signatures. Trends Genet, 2021.

33. Guo, H., et al., Identification of Stemness-Related Genes for Cervical Squamous Cell Carcinoma and Endocervical Adenocarcinoma by Integrated Bioinformatics Analysis. Front Cell Dev Biol, 2021. 9: p. 642724.

34. Pounds, S. and S.W. Morris, Estimating the occurrence of false positives and false negatives in microarray studies by approximating and partitioning the empirical distribution of $p$-values. Bioinformatics, 2003. 19(10): p. 1236-42.

35. Pottoo, F.H., et al., Nanotechnological based miRNA intervention in the therapeutic management of neuroblastoma. Semin Cancer Biol, 2021. 69: p. 100-108.

36. Schmittgen, T.D., Exosomal miRNA Cargo as Mediator of Immune Escape Mechanisms in Neuroblastoma. Cancer Res, 2019. 79(7): p. 1293-1294.

37. Hong, M., et al., Runt-related transcription factor 1 promotes apoptosis and inhibits neuroblastoma progression in vitro and in vivo. J Exp Clin Cancer Res, 2020. 39(1): p. 52.

38. Mus, L.M., et al., The ETS transcription factor ETV5 is a target of activated ALK in neuroblastoma contributing to increased tumour aggressiveness. Sci Rep, 2020. 10(1): p. 218.

39. Boboila, S., et al., Transcription factor activating protein 4 is synthetically lethal and a master regulator of MYCN-amplified neuroblastoma. Oncogene, 2018. 37(40): p. 5451-5465.

40. Schulte, J.H., et al., Transcription factor AP2alpha (TFAP2a) regulates differentiation and proliferation of neuroblastoma cells. Cancer Lett, 2008. 271(1): p. 56-63.

41. Nolan, J.C., et al., A Context-Dependent Role for MiR-124-3p on Cell Phenotype, Viability and Chemosensitivity in Neuroblastoma in vitro. Front Cell Dev Biol, 2020. 8: p. 559553.

42. Qu, $\mathrm{H}$., et al., miRNA-558 promotes tumorigenesis and aggressiveness of neuroblastoma cells through activating the transcription of heparanase. Hum Mol Genet, 2015. 24(9): p. 2539-51.

43. Liu, J.A., et al., Fbxo9 functions downstream of Sox10 to determine neuron-glial fate choice in the dorsal root ganglia through Neurog2 destabilization. Proc Natl Acad Sci U S A, 2020. 117(8): p. 41994210.

44. Hynes-Smith, R.W., et al., Loss of FBXO9 Enhances Proteasome Activity and Promotes Aggressiveness in Acute Myeloid Leukemia. Cancers (Basel), 2019. 11(11). 
45. Yang, L., et al., Engagement of circular RNA HECW2 in the nonautophagic role of ATG5 implicated in the endothelial-mesenchymal transition. Autophagy, 2018. 14(3): p. 404-418.

46. Berko, E.R., et al., De novo missense variants in HECW2 are associated with neurodevelopmental delay and hypotonia. J Med Genet, 2017. 54(2): p. 84-86.

47. Li, X., et al., Mib2 Deficiency Inhibits Microglial Activation and Alleviates Ischemia-Induced Brain Injury. Aging Dis, 2020. 11(3): p. 523-535.

48. Piccolo, P., et al., MIB2 variants altering NOTCH signalling result in left ventricle hypertrabeculation/non-compaction and are associated with Menetrier-like gastropathy. Hum Mol Genet, 2017. 26(1): p. 33-43.

49. Zhang, D., et al., RAB5C, SYNJ1, and RNF19B promote male ankylosing spondylitis by regulating immune cell infiltration. Ann Transl Med, 2021. 9(12): p. 1011.

50. Banh, R.S., et al., PTP1B controls non-mitochondrial oxygen consumption by regulating RNF213 to promote tumour survival during hypoxia. Nat Cell Biol, 2016. 18(7): p. 803-813.

51. Wang, X., et al., RNF213 suppresses carcinogenesis in glioblastoma by affecting MAPK/JNK signaling pathway. Clin Transl Oncol, 2020. 22(9): p. 1506-1516.

52. Liang, C., et al., TRIM36, a novel androgen-responsive gene, enhances anti-androgen efficacy against prostate cancer by inhibiting MAPK/ERK signaling pathways. Cell Death Dis, 2018. 9(2): p. 155.

53. He, J., et al., BTB/POZ zinc finger protein ZBTB16 inhibits breast cancer proliferation and metastasis through upregulating ZBTB28 and antagonizing BCL6/ZBTB27. Clin Epigenetics, 2020. 12(1): p. 82.

54. Xiao, G.Q., et al., ZBTB16 is a sensitive and specific marker in detection of metastatic and extragonadal yolk sac tumour. Histopathology, 2017. 71(4): p. 562-569.

\section{Tables}

Due to technical limitations, tables are only available as a download in the Supplemental Files section.

\section{Figures}


$\mathbf{A}$
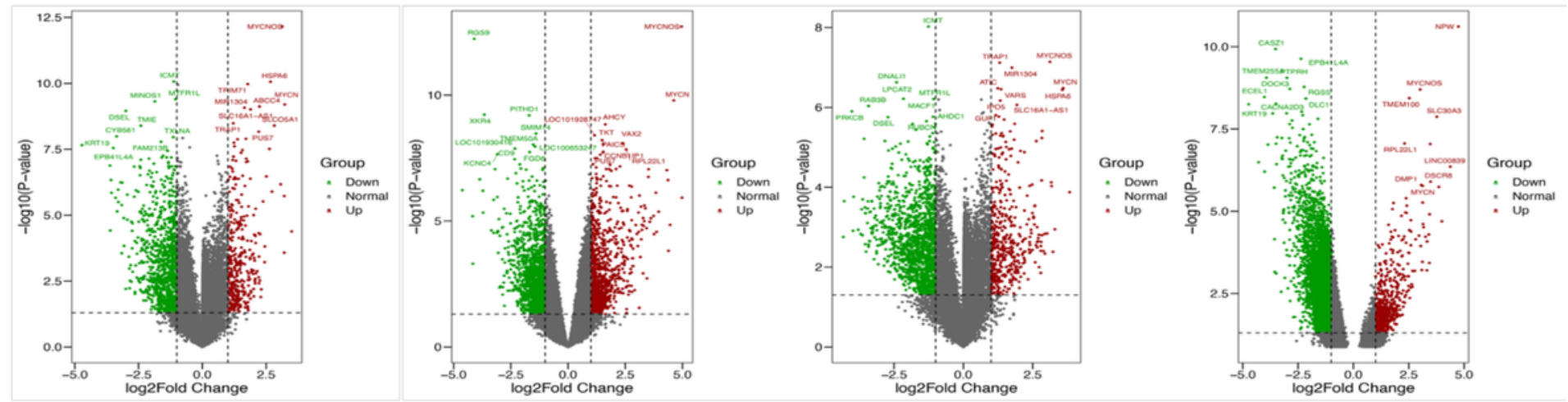

B
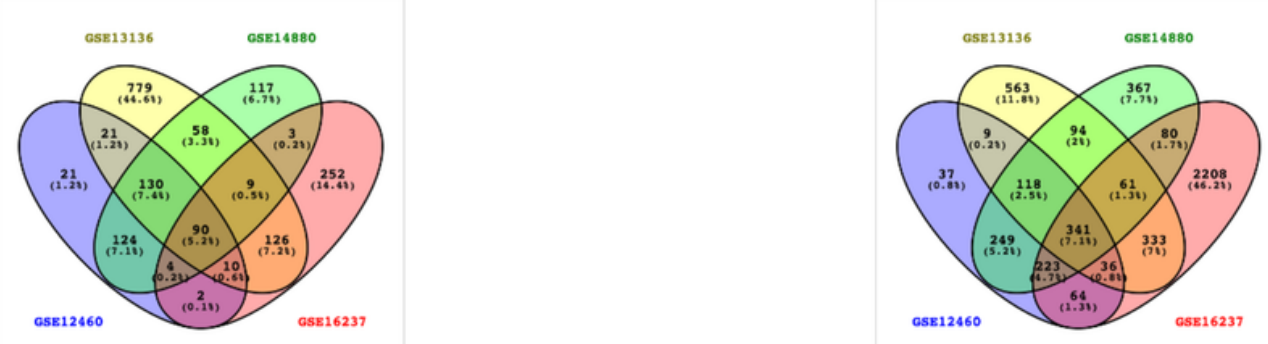

$\mathbf{C}$
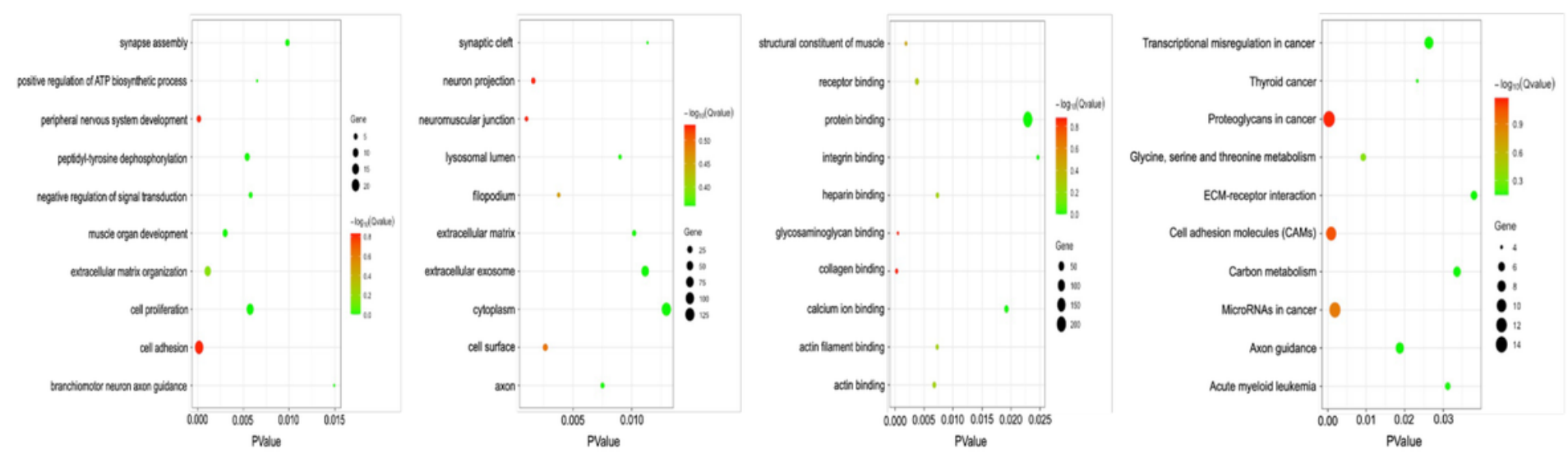

Figure 1

Volcano plots distribution of DEGs, identification of overlapped DEGs, and GO and KEGG enrichment analysis. (A)The microarray datasets of GSE12460, GSE13136, GSE14880, and GSE16237. The green points indicate the screened down-regulated DEGs, the red points indicate the screened up-regulated DEGs, and the gray points indicate genes with no significant differences. All DEGs are screened based on P-value $<0.05$ and | fold change $\mid>1$, (B)Venn diagram of 90 overlapped up-regulated DEGs, and 341 overlapped down-regulated DEGs of four microarray datasets, (C) Analysis of Biological process, Cellular component, Molecular function, and KEGG. (DEGs, Differentially Expressed Genes, GO, gene ontology, KEGG, Kyoto Encyclopedia of Genes and Genomes) 
A

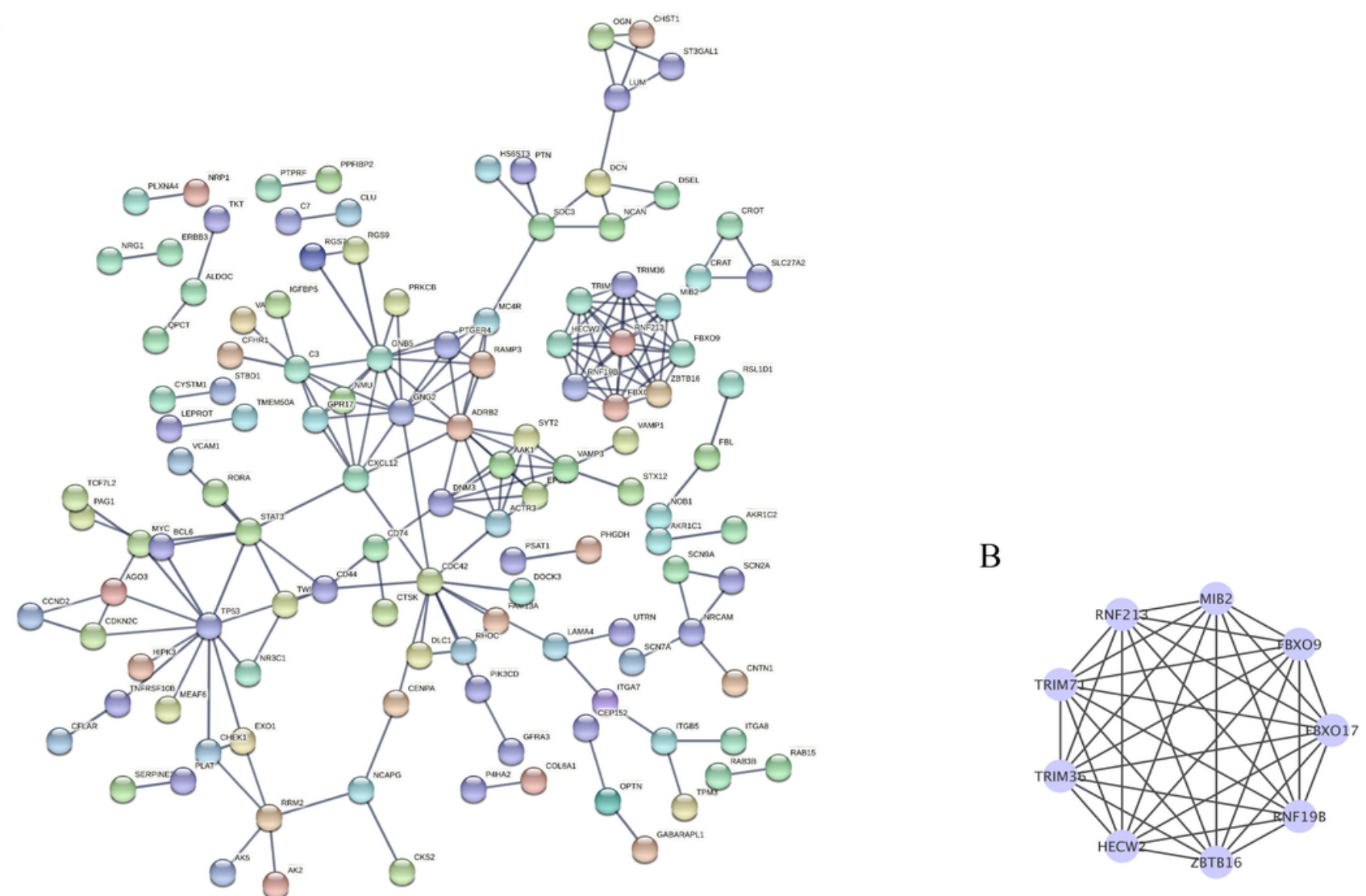

Figure 2

PPI network construction and MCODE analysis. (A) PPI network of DEGs was constructed in STRING. (B) PPI network of DEGs was constructed in Cytoscape and MCODE analysis. (PPI, Protein-Protein Interaction) 
A

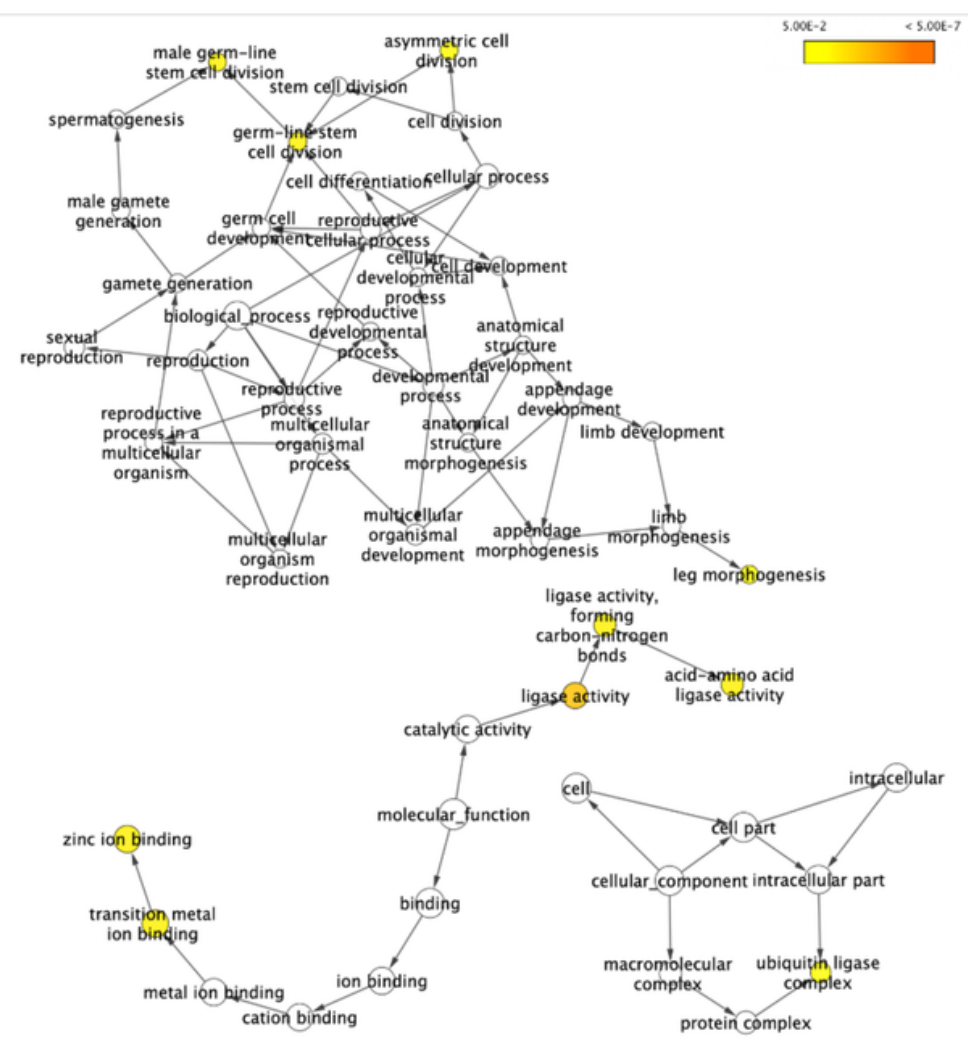

B

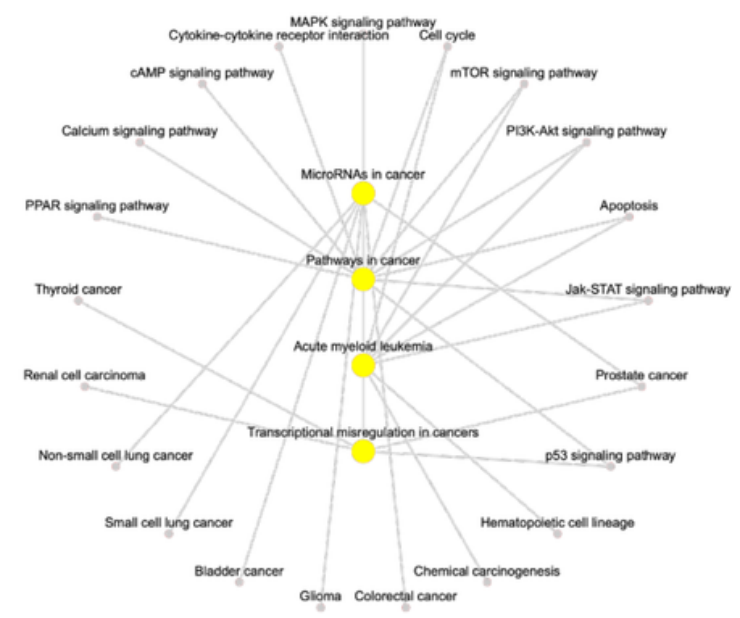

Figure 3

GO and KEGG enrichment analysis of hub module. (A) GO enrichment analysis of the hub module was performed through the BiNGO plug-in. (B) KEGG enrichment analysis of the hub module. Yellow points indicated GO terms and pathways with significant differences $(P<0.05)$. 


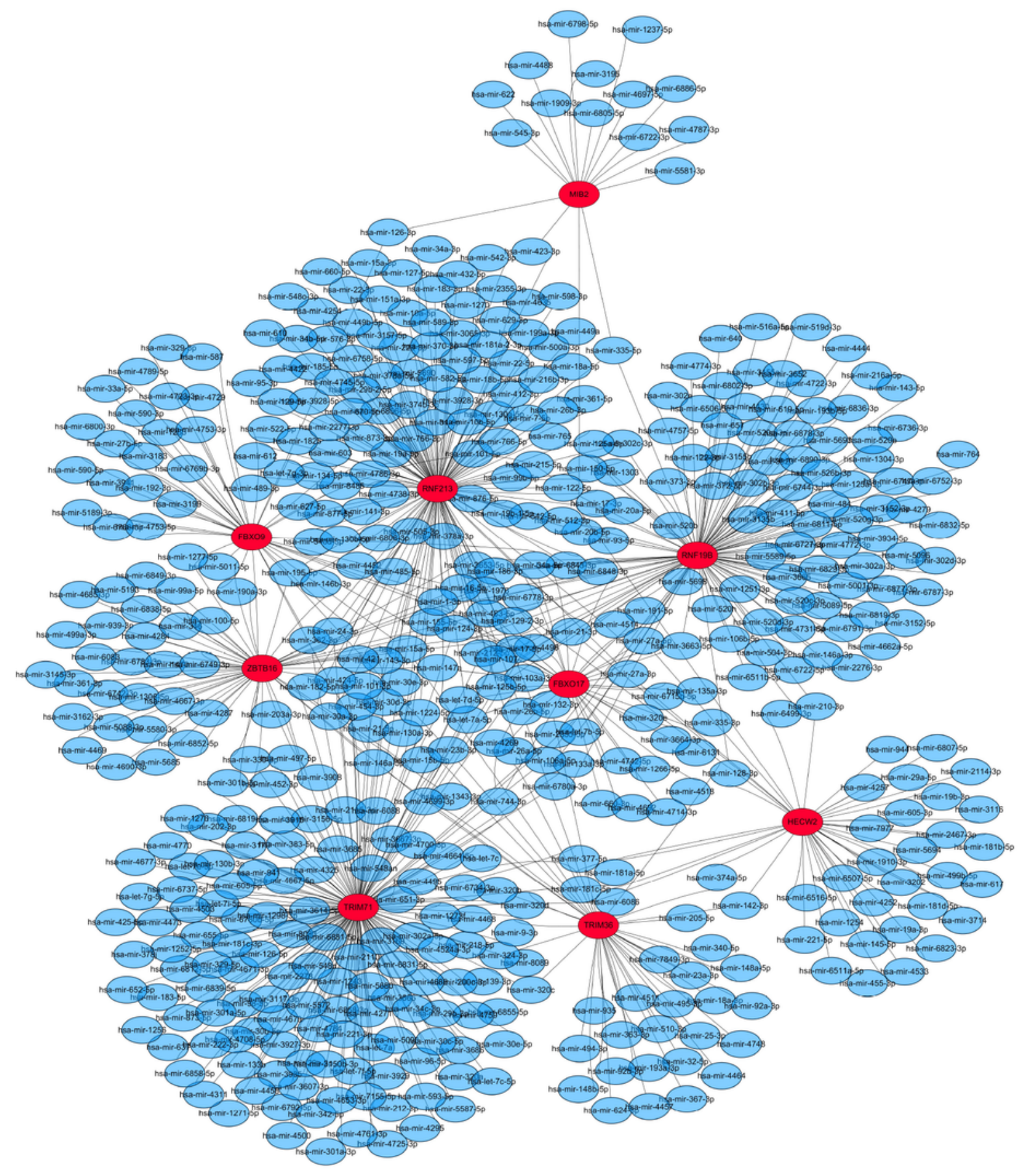

Figure 4

miRNA prediction of target genes. The Red points represent mRNAs, and the blue points represent predicted miRNAs. 


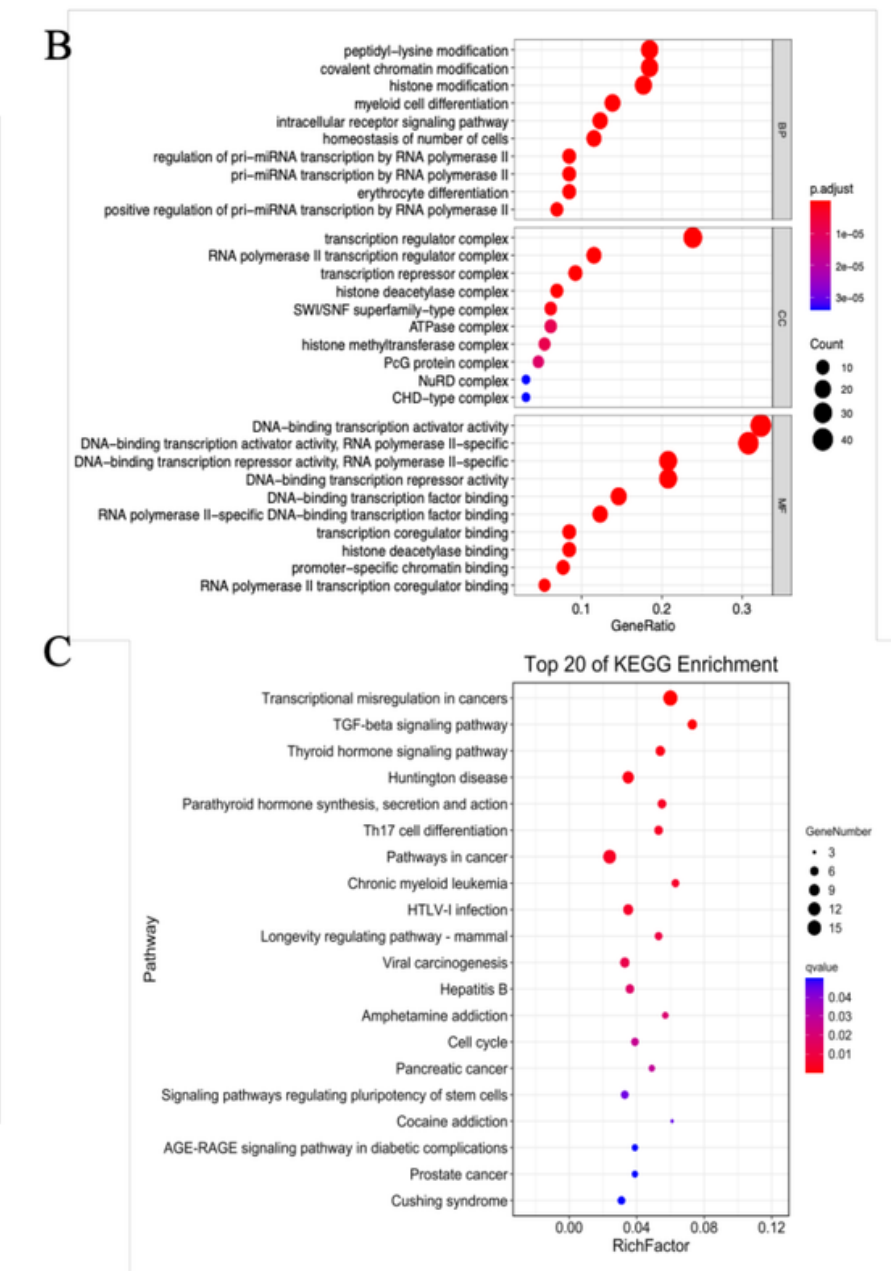

Figure 5

TF prediction of target genes and enrichment analysis. (A) In constructing the mRNA-TF network, the red points represent mRNAs, and the blue points represent predicted TFs. (B) GO analysis of TFs. (C) KEGG analysis of TFs. (TFs, Transcription Factors) 
FBXO9

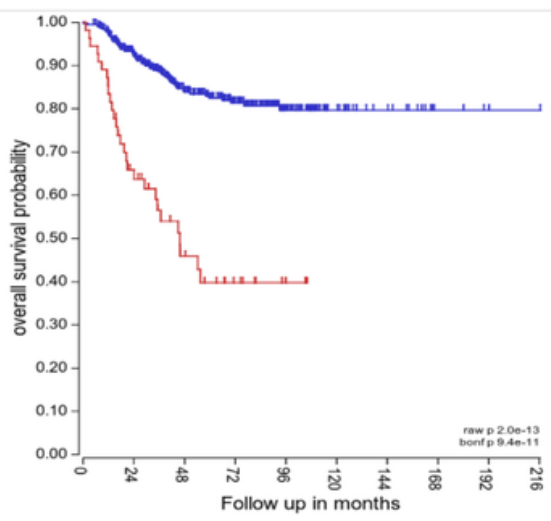

MIB2

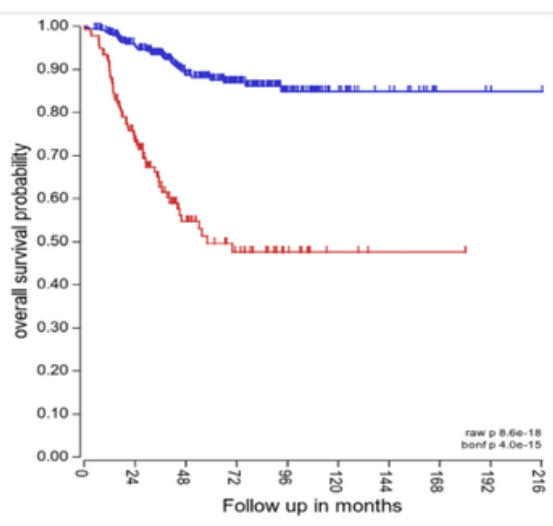

TRIM36

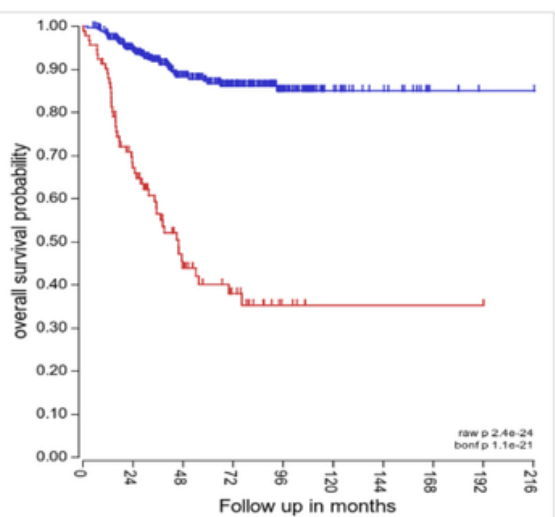

FBXO17

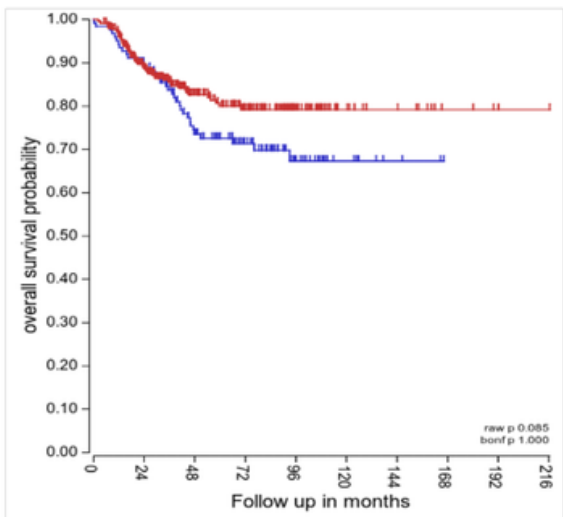

RNF19B

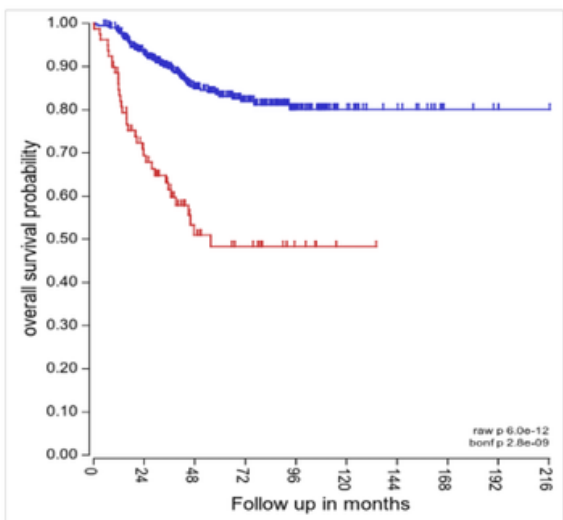

ZBTB16

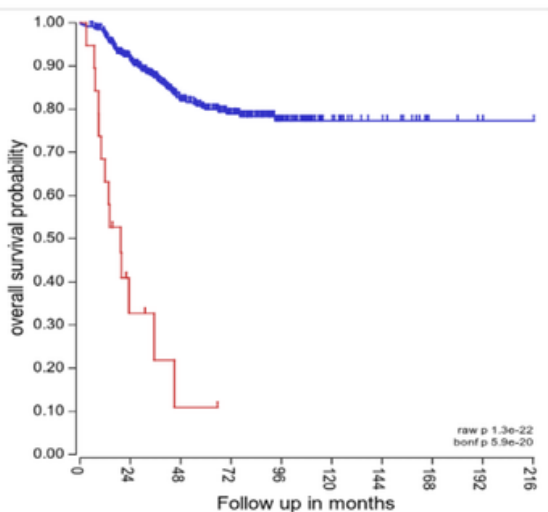

HECW2

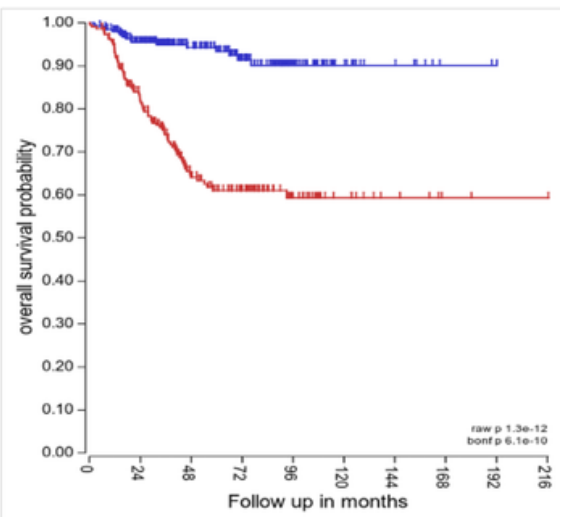

RNF312

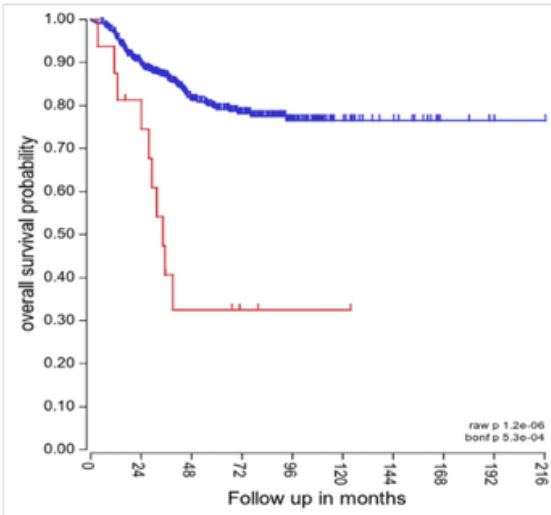

Figure 6

Survival analysis of hub genes. Survival analysis of FBX09, FBX017, HECW2, MIB2, RNF19B, RNF312, TRIM36, and ZBTB16. The blue curves represent the high expression of genes in NB, and the red curves represent the low expression of genes in NB. Bonf $p<0.05$ was regarded as the critical point with statistical significance. 
A
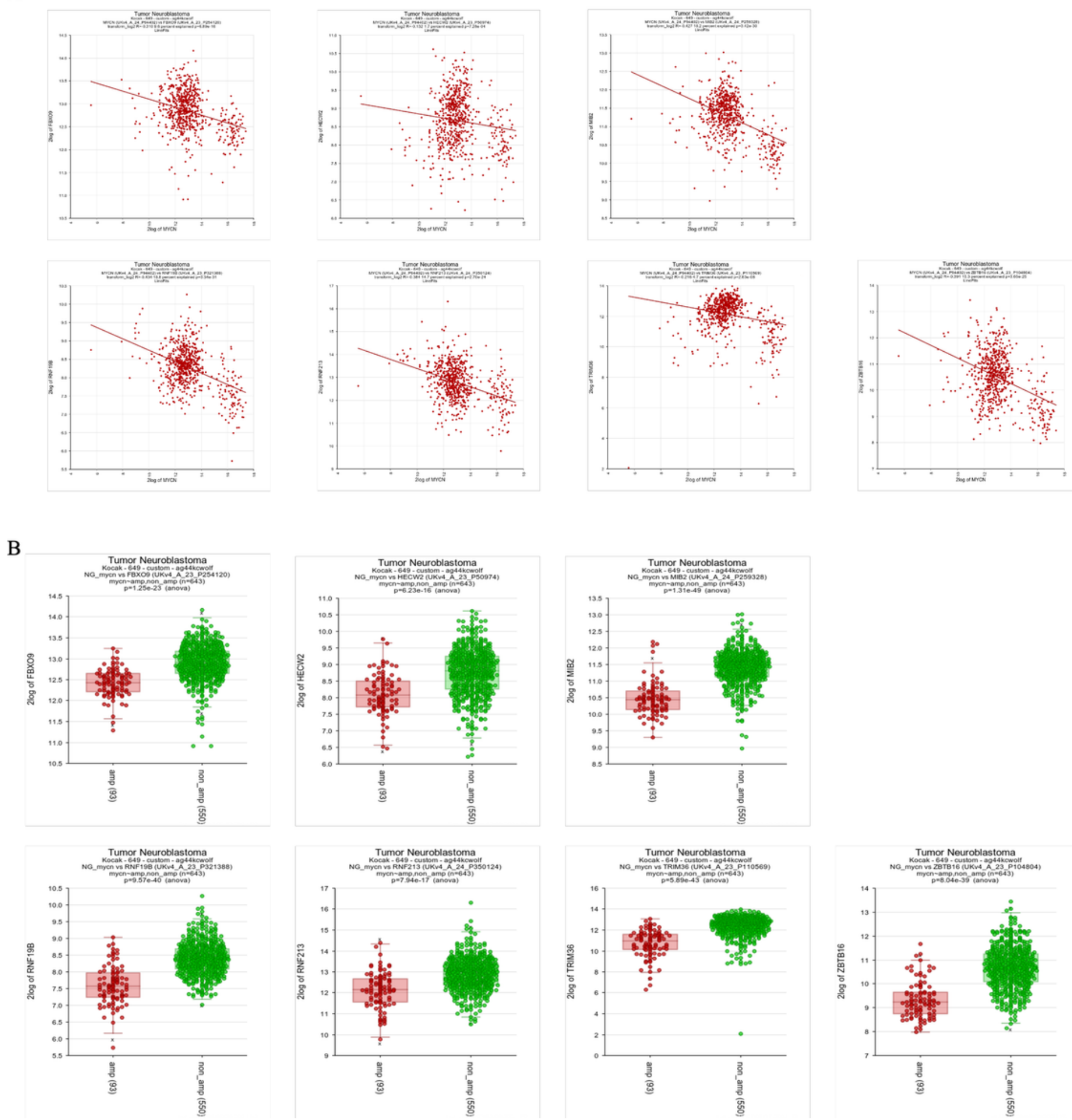

Figure 7

Correlation and expression analysis of hub genes in NB. (A) Correlation analysis shows that hub genes were negatively correlated with MYCN $(P<0.001)$. (B) Hub genes expression was higher in MYCN nonamplified NB cells $(P<0.001)$. 
A

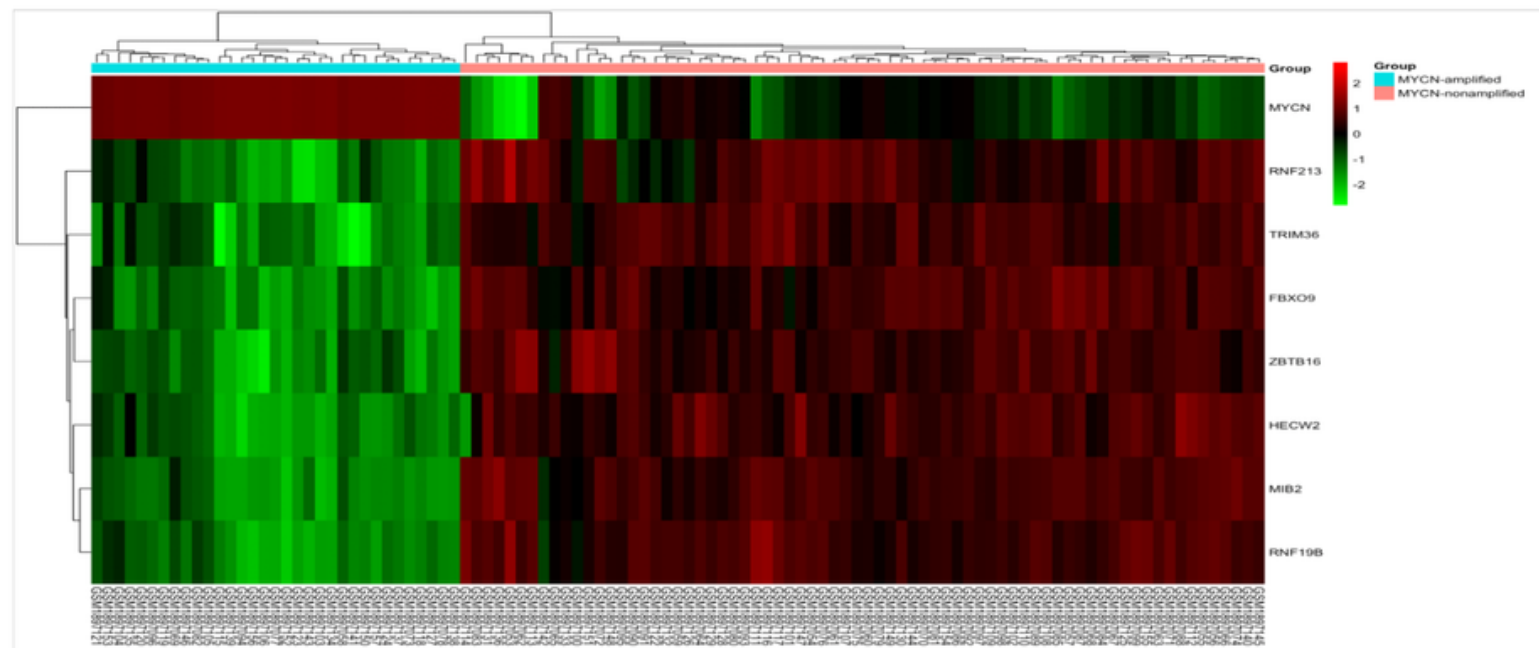

B

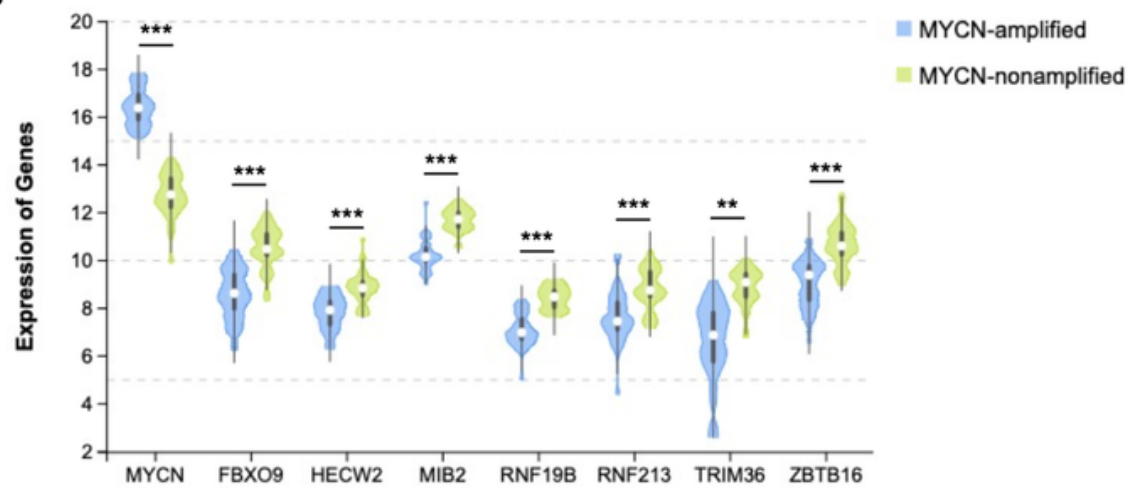

C

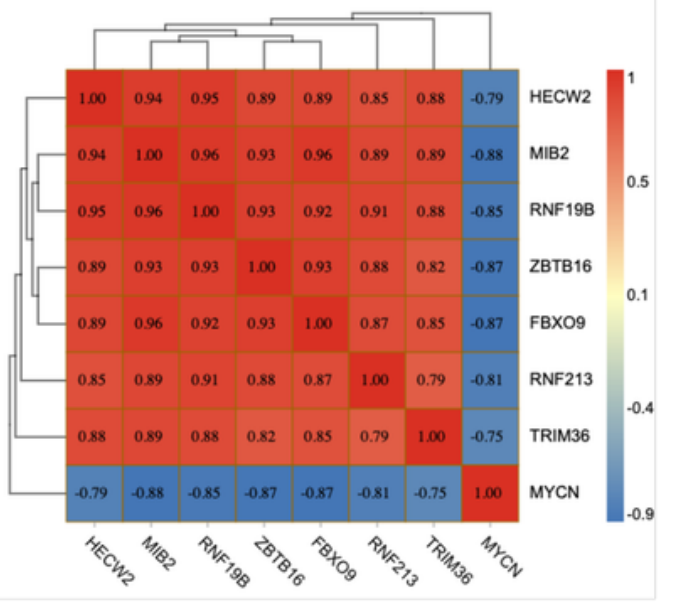

D

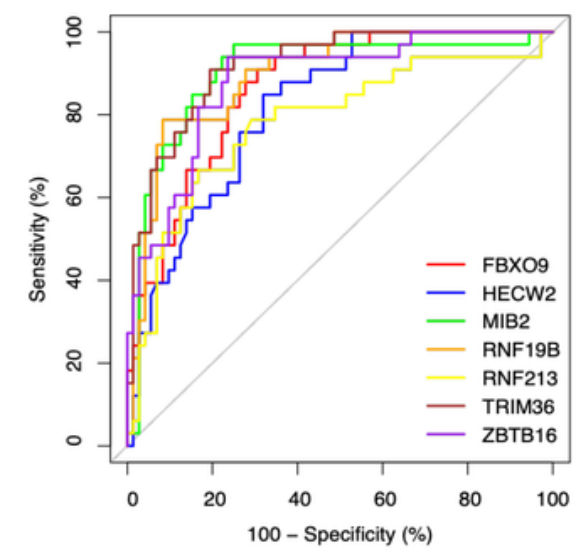

\section{Figure 8}

Validation of hub genes. (A) The GSE73517 dataset was used to validate the expression of hub genes and MYCN, the results of which were presented as a heatmap. (B) Detailed expression of hub genes and MYCN in MYCN amplified and MYCN nonamplified NB cells. (C) The correlation of hub genes and MYCN in NB cells was evaluated. Red: positive correlation, Blue: negative correlation. (D) The GSE73517 dataset was used to validate the diagnostic effectiveness of the biomarkers for NB by ROC analysis. 
A

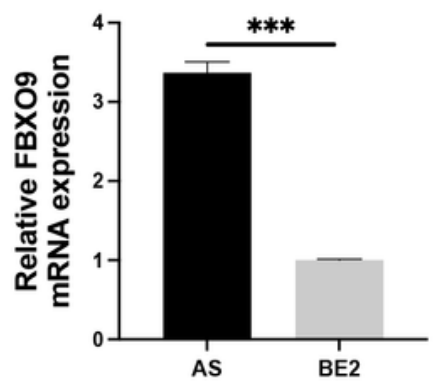

C

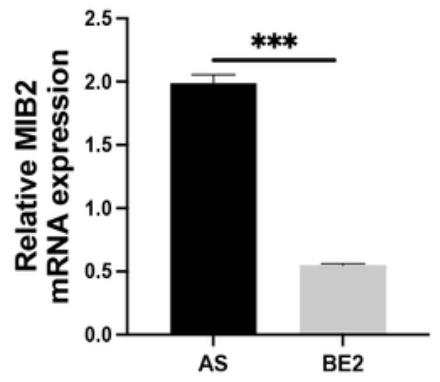

E



G

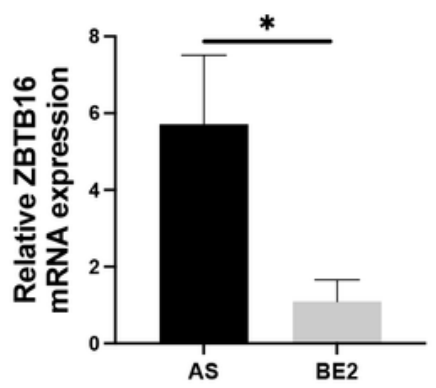

B

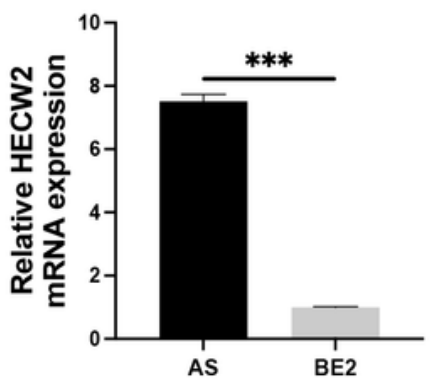

D

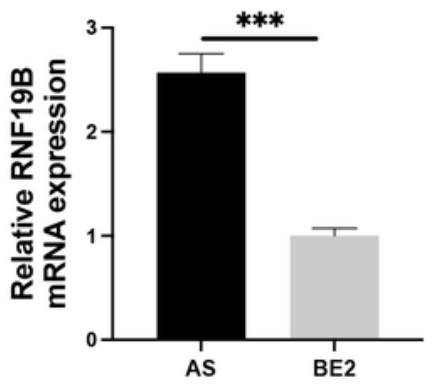

F



Figure 9

The results of hub genes mRNA levels. (A) FBX09, (B) HECW2, (C) MIB2, (D) RNF19B, (E) RNF312, (F) TRIM36, and (G) ZBTB16 mRNA levels were detected in NB (*, P<0.05, **, P<0.01, ***, P<0.001). 
A
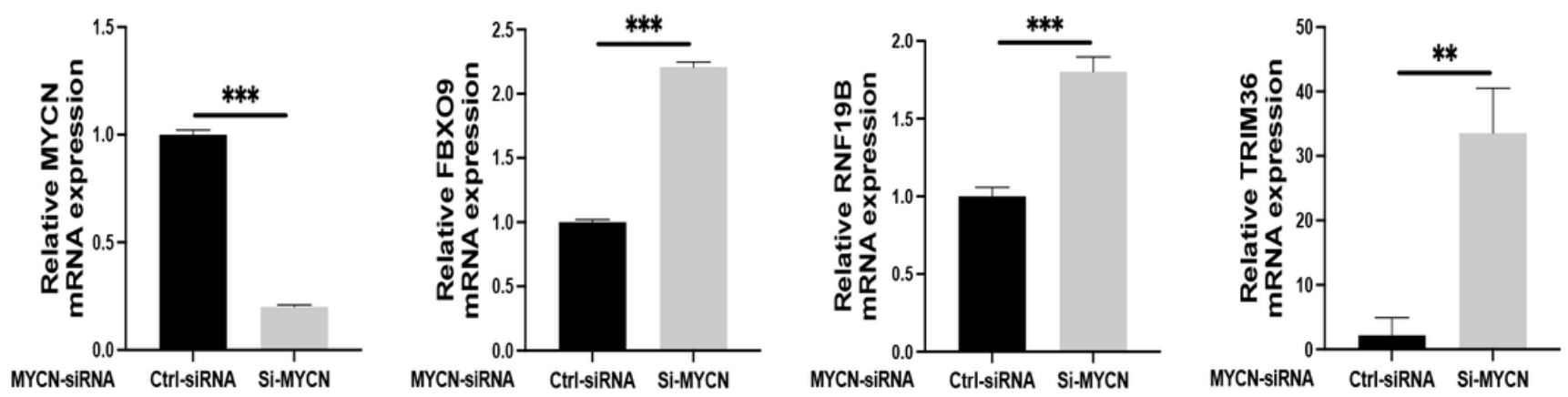

B
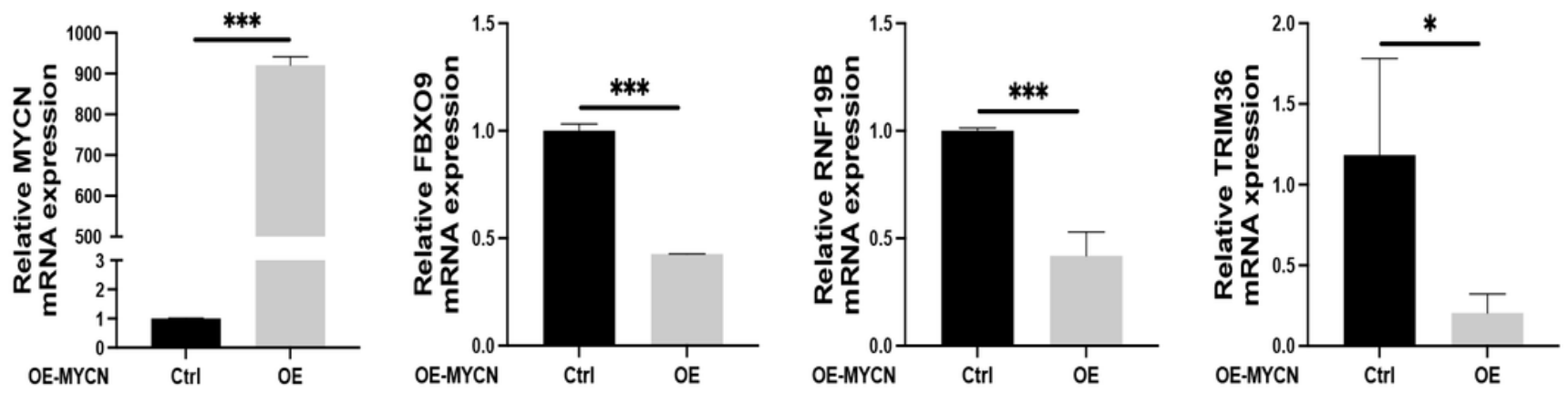

Figure 10

MYCN regulates the expression of FBX09, RNF19B, and TRIM36. The changes of MYCN and hub genes (FBX09, RNF19B, and TRIM36) mRNA levels were detected by RT-PCR after (A) transfected MYCN siRNA into BE2 cells, and (B) transfected MYCN overexpressed plasmid into AS cells $(*, P<0.05, * \star, P<0.01$, $* \star \star$, $P<0.001)$.

\section{Supplementary Files}

This is a list of supplementary files associated with this preprint. Click to download.

- table1.tif

- Table2.tif

- table3.tif 